\title{
$\mathrm{e}-$ Blood
}

\section{Identification of microRNA expression patterns and definition of a microRNA/mRNA regulatory network in distinct molecular groups of multiple myeloma}

\author{
*Marta Lionetti, ${ }^{1}$ *Marta Biasiolo, ${ }^{2}$ Luca Agnelli, ${ }^{1}$ Katia Todoerti, ${ }^{1}$ Laura Mosca, ${ }^{1}$ Sonia Fabris, ${ }^{1}$ Gabriele Sales, ${ }^{2}$ \\ Giorgio Lambertenghi Deliliers, ${ }^{1}$ Silvio Bicciato, ${ }^{3}$ Luigia Lombardi, ${ }^{1}$ Stefania Bortoluzzi, ${ }^{2}$ and Antonino Neri ${ }^{1}$ \\ ${ }^{1}$ Department of Medical Sciences, University of Milan, Hematology 1-Centro Trapianti Midollo Osseo, Foundation Istituto di Ricovero e Cura a Carattere \\ Scientifico Policlinico, Milan; '2Laboratory of Computational Biology, Department of Biology, University of Padua, Padua; and ${ }^{3}$ Department of Biomedical \\ Sciences, University of Modena and Reggio Emilia, Modena, Italy
}

To date, little evidence of miRNA expression/ deregulation in multiple myeloma has been reported. To characterize miRNA in the context of the major multiple myeloma molecular types, we generated miRNA expression profiles of highly purified malignant plasma cells from $\mathbf{4 0}$ primary tumors. Furthermore, transcriptional profiles, available for all patients, were used to investigate the occurrence of miRNA/predicted target mRNA pair anticorrelations, and the miRNA and genome-wide DNA data were integrated in a subset of patients to evaluate the influence of allelic imbalances on miRNA expression. Differential miRNA expression patterns were identified, which were mainly associated with the major IGH translocations; particularly, $t(4 ; 14)$ patients showed specific overexpression of let-7e, miR-125a-5p, and miR-99b belonging to a cluster at $19 q 13.33$. The occurrence of other lesions (ie, 1q gain, $13 q$ and $17 p$ deletions, and hyperdiploidy) was slightly characterized by specific miRNA signatures. Furthermore, the occurrence of several allelic imbalances or loss of heterozygosity was found significantly associated with the altered expression of miRNAs located in the involved regions, such as let-7b at $22 q 13.31$ or $m i R-140-3 p$ at $16 q 22$. Finally, the integrative analysis based on computational target prediction and miRNA mRNA profiling defined a network of putative functional miRNA-target regulatory relations supported by expression data. (Blood. 2009;114:e20-e26)

\section{Introduction}

Multiple myeloma (MM) is a malignant proliferation of bone marrow (BM) plasma cells (PCs), characterized by a profound genomic instability involving both numerical and structural chromosomal aberrations of potential prognostic relevance. ${ }^{1}$ Nearly half of MM tumors are hyperdiploid (HD) with multiple trisomies of nonrandom odd-numbered chromosomes and a low prevalence of chromosomal translocations involving the immunoglobulin heavy chain $(I G H)$ locus at $14 \mathrm{q} 32$ and chromosome 13 deletion $^{1}$; the others are nonhyperdiploid (NHD) tumors often showing chromosome 13 deletion, 1q gain, and $I G H$ translocations, ${ }^{2}$ with the most frequent partners being 11q13, 4p16,16q23, 20q11, and 6p21. The deregulation of at least one of the cyclin D genes is observed in almost all MM cases and, in combination with recurrent $I G H$ translocations, has been proposed for a molecular classification of MM called translocation/cyclin (TC) classification..$^{3,4}$ The occurrence of specific transcriptional patterns associated with the molecular subgroups and major genetic lesions of MM has been extensively described in several studies by us and others. ${ }^{2-10}$

miRNAs are endogenous approximately $22 \mathrm{nt}$ RNAs that play important regulatory roles in animals and plants by targeting mRNAs for cleavage or translational repression. ${ }^{11}$ It has been suggested that chromosomal abnormalities and other types of genetic or epigenetic alterations might contribute to miRNA deregulation in cancer. ${ }^{12,13}$
There are still few published data concerning miRNA expression in MM. Loffler et al have shown that interleukin-6 (IL-6) regulates $m i R$ - 21 transcription in IL-6-dependent human myeloma cell lines (HMCLs) through a STAT-3-related mechanism, and that ectopic miR-21 expression can sustain their growth in the absence of IL-6. ${ }^{14}$ Pichiorri et al have reported a set of miRNAs that can be associated with neoplastic transformation and progression in MM. ${ }^{15}$ Our group has identified a set of deregulated miRNAs in HMCLs that correlate with copy number $(\mathrm{CN})$ alterations or gene expression patterns. ${ }^{16,17}$ More recently, Roccaro et al reported the down-regulation of $m i R-15 a$ and $m i R-16$ in a small cohort of relapsed-refractory $\mathrm{MM}$ patients, showing that they may regulate the proliferation and growth of MM cells in vitro and in vivo. ${ }^{18}$

We here describe the miRNA expression profiling of MMs in the context of the major recurrent molecular alterations of the disease. Furthermore, in an attempt to define the consequences of differential miRNA expression, we searched for functional targeting relationships by the definition of a global miRNA/mRNA regulatory network.

\section{Methods}

\section{Patient samples}

BM aspirates were obtained during standard diagnostic procedures from 54 untreated patients (48 MMs and 6 plasma cell leukemias [PCLs]), most
Submitted August 12, 2009; accepted September 24, 2009. Prepublished online as Blood First Edition paper, October 21, 2009; DOI 10.1182/blood2009-08-237495.

${ }^{*}$ M.L. and M.B. contributed equally to this study.
The online version of this article contains a data supplement.

C 2009 by The American Society of Hematology 
of whom have been included in our previous reports. ${ }^{5,17}$ All patients had given their informed consent in accordance with the Declaration of Helsinki, and all studies were approved by the Institutional Review Board of the Fondazione IRCCS Policlinico of Milan. Normal control samples (NCs) were obtained from 3 healthy donors. Patients had been diagnosed on the basis of previously described criteria. ${ }^{5}$ PCs were purified from BM biopsies using CD138 immunomagnetic microbeads (MidiMACS; Miltenyi Biotec). ${ }^{19}$ The purity of the positively selected PCs ( $\geq 90 \%$ ) was assessed by flow cytometry. Patients have been stratified according to the previously described TC classification. ${ }^{3,4}$ Briefly, the TC1 group included patients characterized by the $\mathrm{t}(11 ; 14)$ or $\mathrm{t}(6 ; 14)$ translocation; TC2, those showing low to moderate levels of the $C C N D 1$ gene in the absence of any primary IGH translocation; TC3, tumors that do not fall into any of the other groups; TC4, cases that show $\mathrm{t}(4 ; 14)$ translocation; and TC5, either the $\mathrm{t}(14 ; 16)$ or $\mathrm{t}(14 ; 20)$ translocations.

\section{miRNA expression profiling}

Thirty-eight MM, 2 PCL, and 3 NC samples were investigated for miRNA expression by microarray analysis. The patient samples were selected to be representative of the 5 TC groups, and the PCL samples with translocated $M A F$ genes were included because of the relatively small number of TC5 MM patients. Fluorescence in situ hybridization information regarding the major numerical chromosomal alterations was already available (supplemental Table 1, available on the Blood website; see the Supplemental Materials link at the top of the online article). The total RNA extraction and quality assessment were performed as previously described. ${ }^{16}$ The samples were profiled on the Agilent Human miRNA Microarray V2 (Agilent Technologies), consisting of 60-mer DNA probes synthesized in situ that represent 723 human and 76 human viral miRNAs from the Sanger database (Version 10.1), using the one-color technique in accordance with the manufacturer's instructions. ${ }^{16}$ The human miRNAs data were reannotated on Sanger Release 12.0 and normalized using the Aroma Light package for Bioconductor. To overcome scaling biases resulting from background subtraction, the data were converted to obtain positive values throughout the dataset, at a minimum value of 1 . The raw and normalized microRNA data are available through GEO accession number GSE17498. Hierarchical agglomerative clustering of the selected probe lists was performed using Pearson correlation coefficient and average linkage as distance and linkage metrics, respectively. ${ }^{19}$ Supervised analyses were carried out using Significant Analysis of Microarrays software, Version 3.02 as previously described. ${ }^{5}$ The significance threshold (at a $q$ value $=0$ ) was determined tuning the $\Delta$ parameter on the false discovery rate and controlling the $q$ value of the selected probes. The cross-validation of the identified signatures was performed using the default options of lda package for Linear Discriminant Analysis in R software, choosing Leave-One-Out Cross-Validation method and applying a 1000-permutation model on a bootstrapped resampled dataset.

\section{Quantitative RT-PCR}

Selected mature miRNAs underwent quantitative reverse-transcribed polymerase chain reaction (RT-PCR) using TaqMan microRNA assays (Applied Biosystems) as previously described. ${ }^{16}$

\section{Gene expression profiling}

The transcriptional profiles of the patients have been generated using Affymetrix GeneChip HG-U133A arrays as previously described ${ }^{5,20}$ and are available through GEO accession number GSE13591. The raw intensity signals were extracted from CEL files and normalized using the RMA "function of affy" package for Bioconductor and custom GeneAnnot-based Chip Annotation Files, Version 2.0.1 (CDF) ${ }^{21}$ (available at http:// www.xlab.unimo.it/GA_CDF), thus leading to the definition of 12195 unique well-characterized genes.

\section{Genome-wide profiling}

Nineteen of the $40 \mathrm{MM} / \mathrm{PCL}$ samples profiled for miRNA expression underwent genome-wide DNA analysis using Affymetrix GeneChip Human

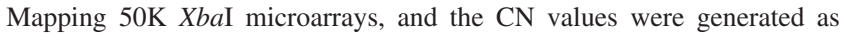

recently described. ${ }^{20}$ Briefly, raw $\mathrm{CN}$ values for individual SNPs and the probability of loss of heterozygosity ( $\mathrm{LOH})$ were extracted from CEL files and converted into signal intensities using Affymetrix GTYPE 4.1 and CNAT 4.0.1 software; robust estimate of genotype calling was performed using BRLMM distance classifier. Piecewise constant estimates of the underlying local DNA CN variation were calculated using the DNAcopy Bioconductor package, which looks for optimal breakpoints using circular binary segmentation, and the estimated profiles were normalized using the self-developed FBN package for R software. LOH was defined applying a 100-kb smoothing window (ie, 2-fold the mean intermarker distance on the arrays) on the CNAT-derived value with a probability higher than 0.95 for the detection of a monoallelic polymorphism. ${ }^{20}$ The CEL files are available through GEO accession number GSE16121.

\section{miRNA target prediction}

miRNA targets have been predicted applying miRanda algorithm ${ }^{22}$ on human miRNA and transcript sequences of miRBase Release $12.0^{23}$ and ENSEMBL Release 52, respectively. The score threshold of miRanda, associated with each predicted miRNA-transcript targeting relationship and depending on the sequence alignment and thermodynamic stability of the RNA duplex, has been set at 160. Finally, the correspondence between ENSEMBL_transcript and EntrezGene_ID was defined.

\section{Integrative analysis of miRNA and gene expression profiles to reconstruct a miRNA/gene regulatory network}

The posttranscriptional regulatory network of miRNA and genes in MM has been defined as a directed, bipartite graph in which miRNA-mRNA relationships are supported by both targeting predictions and expression data. Specifically, the network has been reconstructed using the subset of miRNAs and genes characterized by (1) a regulatory relationship according to miRanda predictions and (2) expression profiles strongly anticorrelated. Indeed, because miRNAs tend to down-regulate target mRNAs, the expression profiles of genuinely interacting pairs are expected to be anticorrelated. Thus, for each miRNA/gene pair scored as potentially interacting on the basis of miRanda prediction, the Pearson correlation coefficient of the respective expression vectors in MM samples was calculated and used as an estimator of the functional activity of the miRNAs on the target genes. Genes were considered genuine miRNA targets when included within the top 3\% of all pairs ordered on their anticorrelation score. ${ }^{24}$ This selection gave rise to the final adjacency matrix $S$ of regulatory relations supported by MM expression data and the associated miRNAs/ genes regulatory network. The matrix $S$ defined a bipartite directed network with 2 types of nodes (miRNAs and mRNAs) connected by directed edges, each representing a probable functional regulatory effect of a miRNA on a target gene. The posttranscriptional network allows identifying groups of genes regulated by the same miRNA/s, and of miRNAs regulating specific groups of genes of functional relevance. The networks were drawn using Cytoscape, ${ }^{25}$ and the functional enrichment analysis was performed using Database for Annotation, Visualization and Integrated Discovery 2008 Tool (http://david.abcc.ncifcrf.gov/).

\section{Statistical analysis}

Conventional statistical procedures were applied using standard packages for R software (Kendall $\tau$ correlations, Wilcoxon rank-sum tests, $t$ tests, and Fisher exact tests).

\section{Results}

\section{Global miRNA expression profiling in MM patients}

MiRNA profiles were analyzed by high-density microarrays, specific for 723 human miRNAs, in 40 patients representative of the 5 TC groups (supplemental Table 1), and in PCs from 3 NCs. To determine whether global miRNA profiling could distinguish the molecular groups, we performed an unsupervised analysis using 

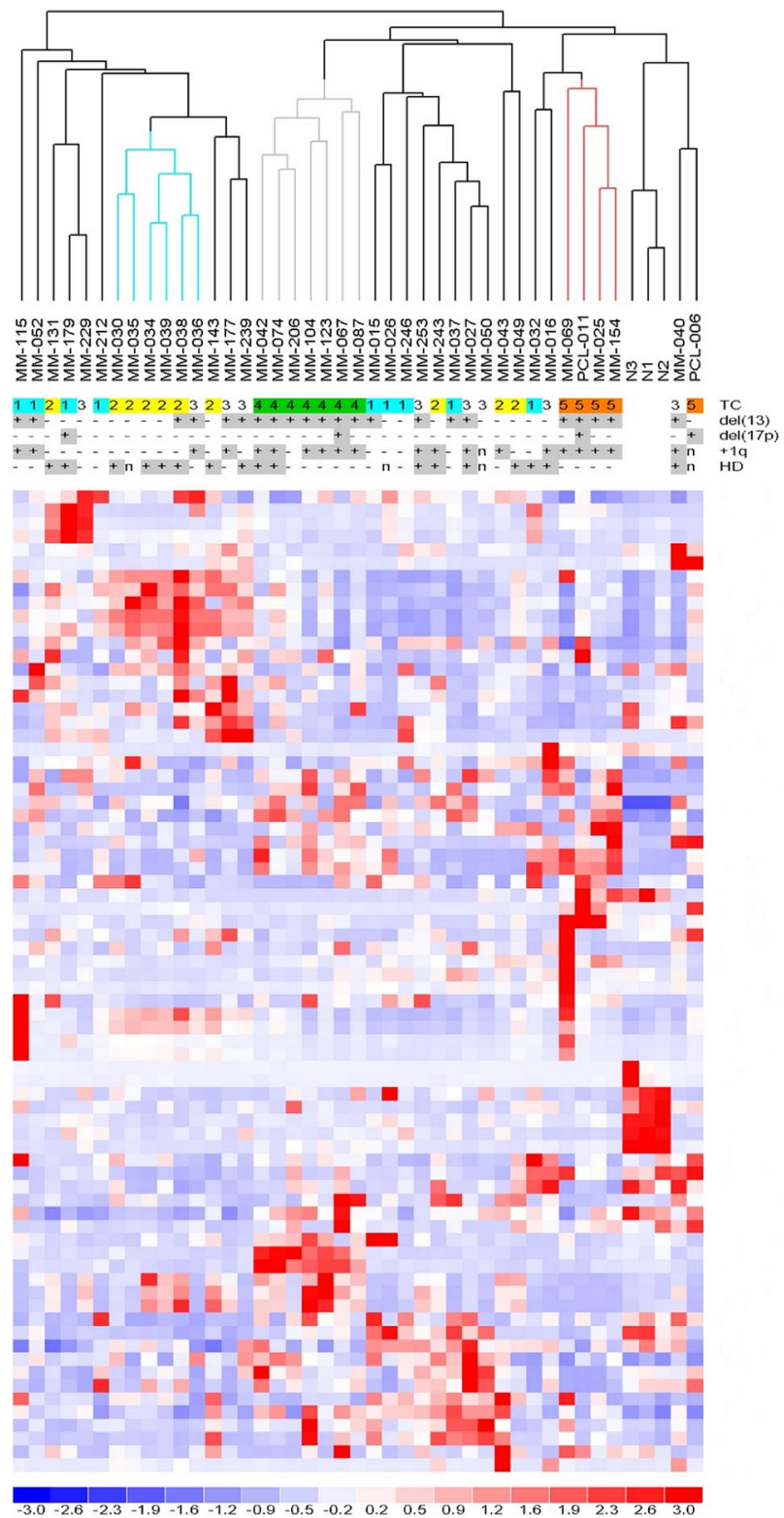

Figure 1. Unsupervised analysis of miRNA expression profiles. Hierarchical clustering of the samples using the 74 most variable miRNAs (patients in columns, miRNAs in rows). The color scale bar represents the relative miRNA expression changes normalized by the standard deviation. The patients' molecular characteristics are shown above the matrix; $n$ indicates unavailable information. Specific characteristics are enriched in colored sub-branches.

conventional hierarchical agglomerative clustering: the 43 samples were described by 74 miRNAs whose average change in expression levels varied at least 2-fold from the mean across the dataset. ${ }^{19}$ The most striking finding was that all of the TC4 patients were tightly clustered (Figure 1 , gray cluster, $P<.001$ ), as were 4 of 5 TC 5 cases (red cluster, $P<.001$ ). The TC2 cases were partially grouped (blue cluster, $P<.005$ ), whereas the TC1 and TC3 samples were scattered along the dendrogram. The NCs were clearly grouped in a distinct sub-branch.

Next, multiclass analysis allowed identifying a set of 26 miRNAs showing highly significant differential expression (at $q$ value $=0$ ) across the 5 TC groups (supplemental Table 2). As shown in Figure 2A, all of the TC groups except TC 3 were characterized by the up-regulation of specific miRNAs. In particular, 10 (38\%) miRNAs (miR-150, miR-133b, miR-99a, miR-133a, miR-155, miR-125b, let-7c, miR-1,
A

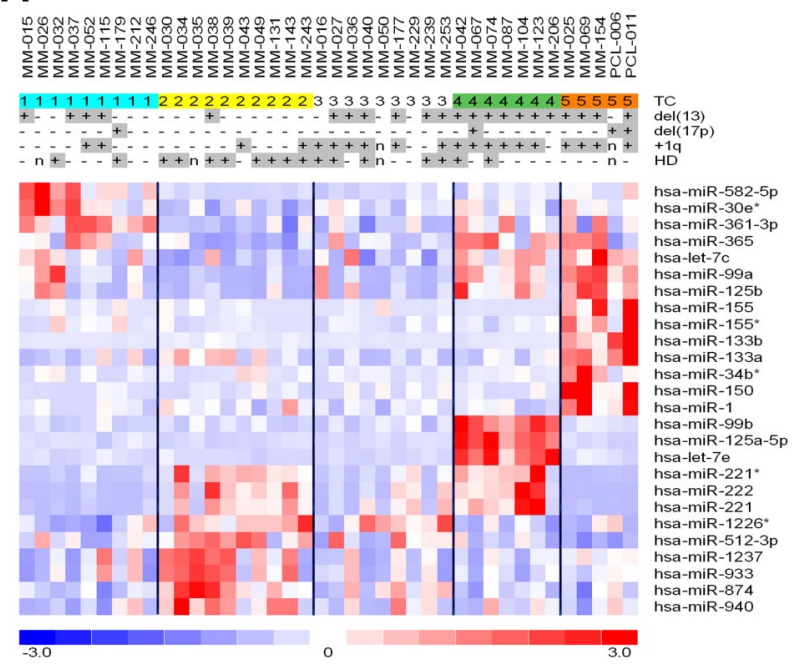

B

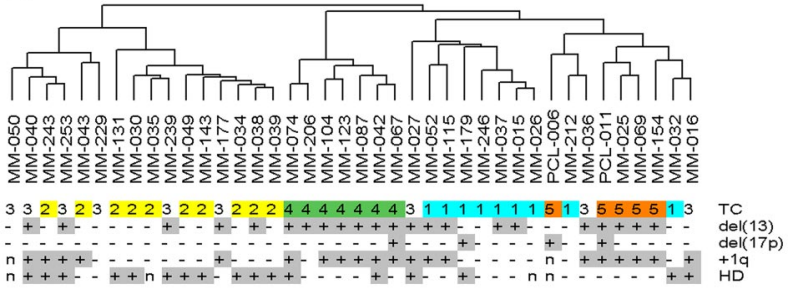

Figure 2. Identification of miRNA signatures characterizing TC classes. (A) Heatmap of the differentially expressed miRNAs in MM patients stratified into the 5 TC groups. (B) Dendrogram of the $40 \mathrm{MM}$ samples clustered according to the expression profiles of the 26 miRNAs.

$m i R-155^{*}$, and $\left.m i R-34 b^{*}\right)$ were expressed at higher levels in TC5 than in the other classes, 7 (27\%) in TC4 (miR-125a-5p, let-7e, miR-99b, $m i R-222, m i R-221, m i R-221^{*}$, and $\left.m i R-365\right), 6(23 \%)$ in TC2 ( $m i R-874$, $m i R-1237, m i R-512-3 p, m i R-940, m i R-933$, and $\left.m i R-1226^{*}\right)$, and $3(11 \%)$ in $\mathrm{TC} 1$ ( $m i R-361-3 p, m i R-582-5 p$, and $\left.m i R-30 e^{*}\right)$. Notably, $m i R-125 a-5 p$, let-7e, and miR-99b, which were associated with the highest scores in the supervised analysis and overexpressed specifically in the TC4 samples, belong to a cluster at 19q13.33, whereas mir-99a, let-7c, and mir-125b-2, highly expressed in the TC5 cases, belong to a paralogous cluster at 21q21.1. Figure 2B shows the $40 \mathrm{MM}$ samples clustered according to the expression profiles of the 26 miRNAs, suggesting their capacity to drive TC distribution into separate branches. To test the correctness of the obtained signature and its capability to discriminate the 5 TC groups, we additionally tested the predictive power of the 26 miRNAs using linear discriminant analysis for classification of multivariate observations. The procedure led to confirm the accuracy of the identified signature, at a percentage of an overall classification rate of $89.3 \%$ : specifically, all TC1, TC4, and TC5 samples were classified correctly, whereas TC2 and TC3 cases showed a misclassification error of $32.8 \%$ and $11.1 \%$, respectively.

We also investigated the differential miRNA expression on the basis of the occurrence of other recurrent chromosomal alterations, such as $1 \mathrm{q}$ gain and $13 \mathrm{q}$ and $17 \mathrm{p}$ deletions, and identified several differentially expressed miRNAs, none of which was located in the involved chromosomal region. Similarly, comparison of the HD and NHD cases revealed a set of up-regulated miRNAs only in the latter. Some of the miRNAs identified in these analyses were the same as those found in $I G H$-translocated patients, in all likelihood because of their representativeness within 1q gain, del(13), del(17), and NHD cases (Figure 3). 

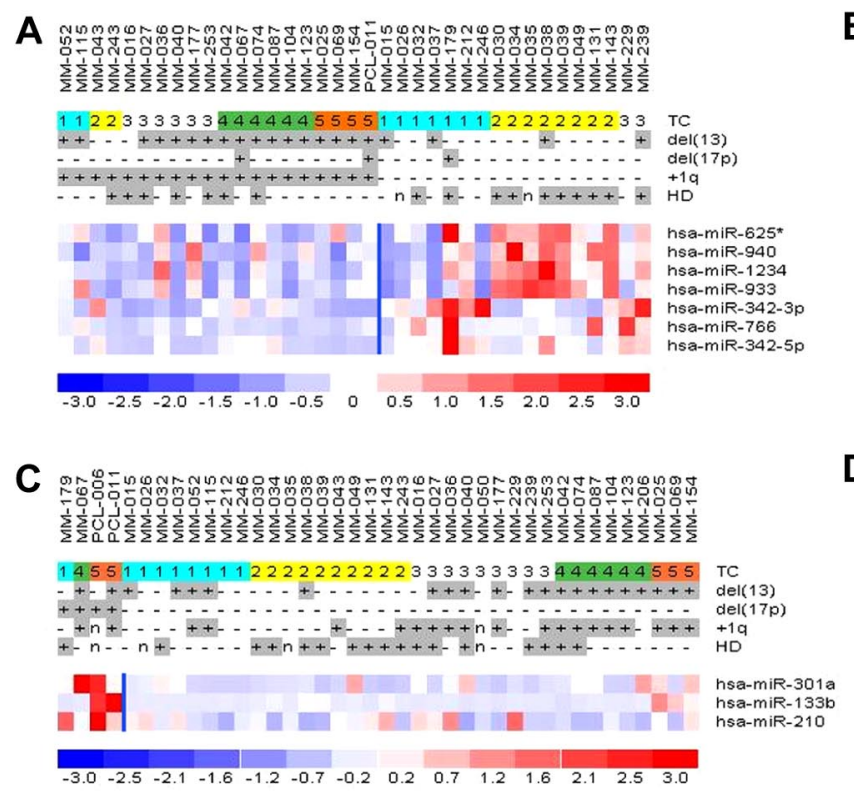

Figure 3. Identification of miRNA signatures characterizing distinct MM genetic subgroups. Supervised analyses identifying the miRNAs that are differentially expressed in MM patients harboring: (A) gain/amplification of the 1q arm, (B) del(13q14), (C) deletion of 17p, and (D) hyperdiploidy.

\section{Quantitative RT-PCR validation of differentially expressed miRNAs}

To validate the microarray data, the levels of some miRNAs (miR-99b, miR-582-5p, miR-155, miR-30e*, miR-125b, miR-133b, $m i R-221, m i R-222, m i R-125 a-5 p$, and $m i R-99 a)$ were quantified by quantitative RT-PCR in all of the 43 samples. As shown in supplemental Table 3, linear correlation analysis indicated a very good correspondence between the 2 techniques.

Furthermore, the expression of $m i R-99 b, m i R-582-5 p, m i R$ $133 b$, and $m i R-125 a-5 p$ was also evaluated in 14 additional cases, 4 cases of which carrying $\mathrm{t}(11 ; 14), 7 \mathrm{t}(4 ; 14)$, and $3 \mathrm{t}(14 ; 16)$ or $\mathrm{t}(14 ; 20)$. Overall, in the 54-sample panel, miR-99b and $m i R$ $125 a-5 p$ were confirmed as showing statistically significant and specific overexpression in the $\mathrm{t}(4 ; 14)$ group; $m i R-133 b$ in the $\mathrm{t}(14 ; 16) / \mathrm{t}(14 ; 20)$ group; and $m i R-582-5 p$ in the $\mathrm{t}(11 ; 14)$ group $(P<.001$ for all miRNAs; Figure 4$)$.

\section{Integrative analysis of miRNA expression profiles with genome-wide copy number variations and occurrence of LOH}

To evaluate the influence in miRNA expression of the allelic imbalances detected at a genome-wide level, we performed an integrative analysis of mature miRNA expression levels and their inferred DNA CN values, available in 19 of 40 cases (supplemental Table 1). ${ }^{20}$ The correlation analysis identified 49 mature miRNAs $(P<.05$; supplemental Table 4$)$, mainly localized on chromosome 1 (8 of 49,16\%), followed by chromosome 19 (6 of 49, 12\%), chromosome 17 (4 of 49, 8\%), and chromosomes 3, 5, 11, 13, 14, and 15 (3 of 49 each, 6\%). Notably, miR-520a-5p, miR-518d-5p, $m i R-498$, and $m i R-520 \mathrm{~g}$ belong to a cluster at $19 \mathrm{q} 13.41$, and $m i R-17, m i R-20 a$, and $m i R-20 a^{*}$ belong to the mir-17 92 cluster at $13 \mathrm{q} 31.3$.

Next, we tested the correlation between miRNA expression and the occurrence of $\mathrm{LOH}$, as defined in our previous report. ${ }^{20} \mathrm{We}$ identified the down-regulation of 14 miRNA genes in the presence of LOH (supplemental Table 5): let-7b, mapped to 22q13.31 $(P=.017)$, and $m i R-662(16 \mathrm{p} 13.3, P<.012)$ were the most
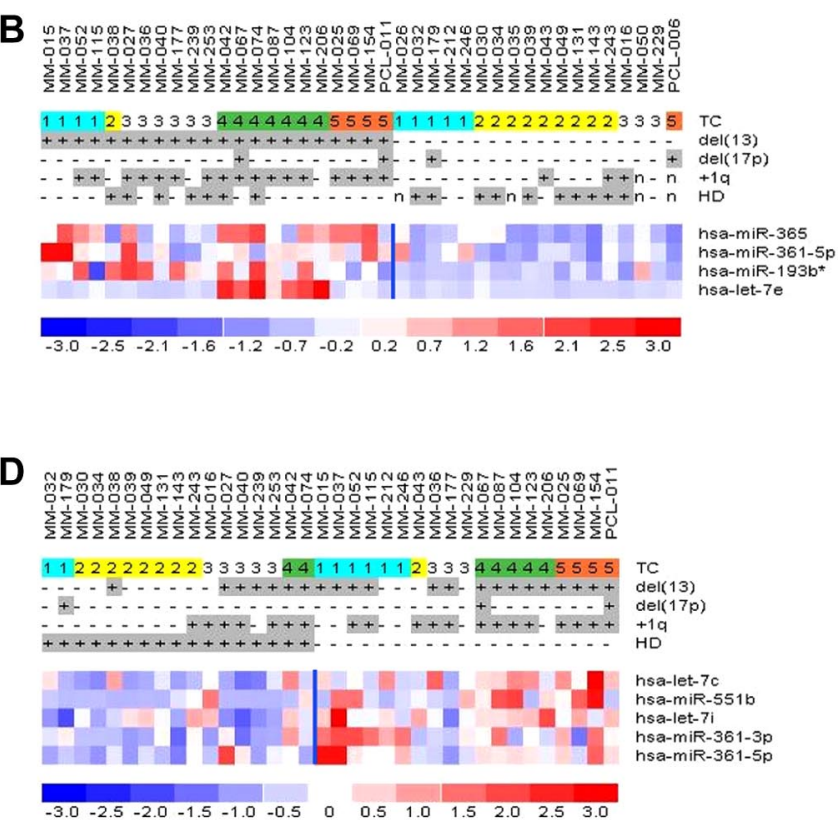

statistically significant. We also highlighted the intronic miRNA $m i R-140-3 p(P=.022)$, which maps to $16 \mathrm{q} 22.1$; the $m i R-19 b$ and miR-20a belonging to the mir-17 92 cluster at 13q31.3 $(P<.022$ and .029 , respectively); and miR-137 $(P<.024)$, which maps to $1 \mathrm{p} 21.3$.

\section{Integrative analysis of miRNA/mRNA expression and reconstruction of a regulatory network in $\mathrm{MM}$}

The integrative analysis of miRNA/mRNA expression profiles allows reconstructing a network of functional interactions occurring in MM from the panel of potential regulatory relationships predicted from sequence information. Our integrative approach assumes that the final effect of a truly functional interaction between a miRNA and its predicted mRNA targets can be seen as a pair of anticorrelated expression profiles. Thus, the set of MiRanda predicted targeting relationships was refined selecting those more strongly supported by the miRNA and mRNA expression data. The entire procedure led to the identification of 23729 regulatory relationships, namely, anticorrelation, involving 628 miRNAs and 6435 predicted target genes, as approximately $47 \%$ of the genes associated with an expression profile were not targets of any of the considered miRNAs and 93 miRNAs (13\%) were not significantly anticorrelated with any target gene. The data from the integrative analysis were used to reconstruct a bipartite direct miRNAs/ mRNAs regulatory network. The number of target genes per miRNA ranged from 1 to 440 (average, 34; mean, 3.7 miRNAs per gene; supplemental Table 6).

Various subnetworks can be derived from the global identified network, as those accounting for the targeting relationships of specific miRNA signatures associated with distinct TC groups. Supplemental Figure 1 reports the subnetwork of the $t(4 ; 14)$ miRNA signature, ie, one of the most consistent and specific subgroups of our dataset. The network of $t(4 ; 14)$ consists of 7 miRNAs and 289 anticorrelated targets, with the number of targets per miRNA ranging from 1 to 113, and approximately $29 \%$ of the genes being targeted by at least 2 miRNAs. Interestingly, 

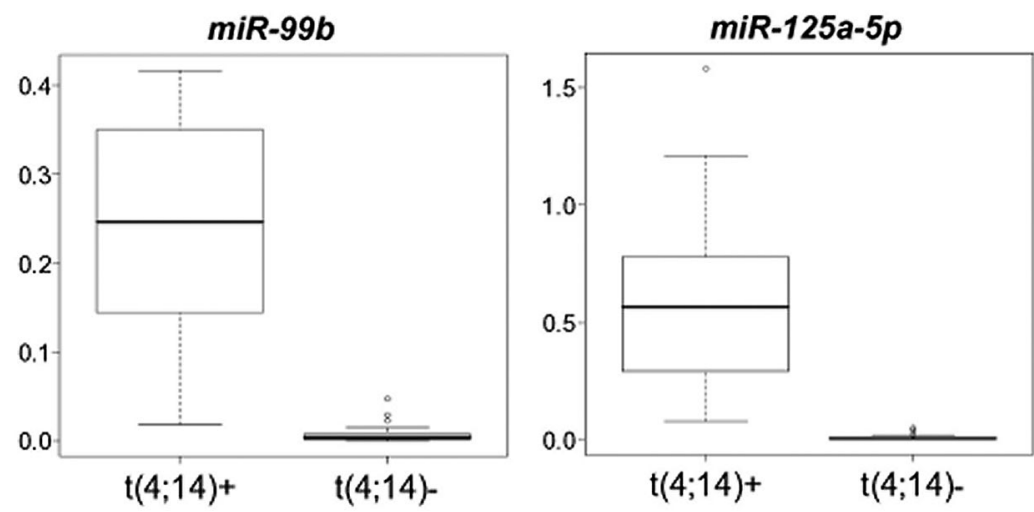

Figure 4. Quantitative RT-PCR validation of miRNA expression. Box plots of miRNAs quantified in quantitative RT-PCR in the broadened panel of $54 \mathrm{MM}-\mathrm{PCL}$ cases, whose expression significantly correlated with the presence of a specific IGH translocation. Expression levels are given as $2^{-\Delta C t}$.

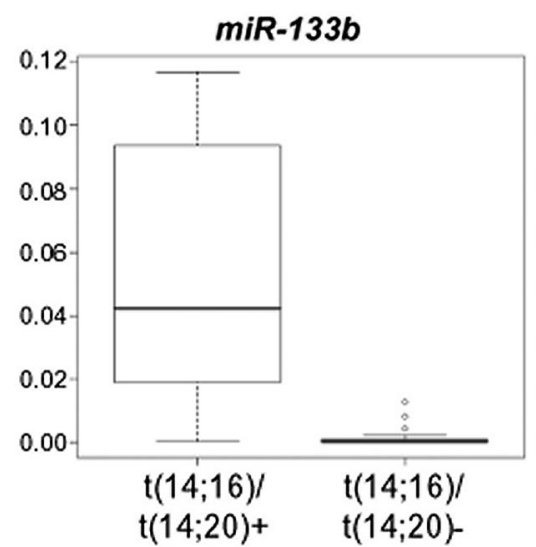

3 genes are commonly regulated by 5 miRNAs (CBFA2T2, core-binding factor, runt domain, $\alpha$ subunit 2, translocated to, 2; $P P P 1 R 16 B$, protein phosphatase 1, regulatory inhibitor subunit 16B; and GOSR2, Golgi SNAP receptor complex member 2). Functional enrichment analysis of anticorrelated targets revealed various overrepresented biologic processes, including chromosome segregation, protein polyubiquitination, cell cycle regulation, and unfolded protein response (supplemental Table 7). Interestingly, a comparison between the identified supported miRNA target genes and mRNAs down-regulated in the $t(4 ; 14)$ cases (as identified in a larger proprietary dataset of $132 \mathrm{MMs}$, data not shown) highlighted that the miRNA targets were significantly enriched in downregulated genes $(P<.001$; supplemental Table 8). Similarly, subnetworks were derived considering the sets of miRNAs differentially expressed in TC5 and TC1 groups (supplemental Table 9).

\section{Discussion}

In this study, the integrative analysis of the different types of genomic data (ie, miRNA and mRNA expression levels and genome-wide $\mathrm{CN}$ profiles) allowed the definition of distinct patterns of miRNA deregulation and the prediction of the miRNA/ mRNA regulatory networks in molecular subtypes of MM.

Particularly, our results highlighted that specific patterns of miRNA expression may differentiate MMs with distinct and well-known genetic alterations. Specific signatures were found to be associated with $\mathrm{t}(4 ; 14)$ or translocated $M A F$ genes and, to a lesser extent, with $\mathrm{t}(11 ; 14)$ and TC2 group (expressing moderate levels of CCNDI in the absence of $I G H$ translocation). This scenario is reminiscent of that previously described at mRNA level in the context of the TC classification. ${ }^{4}$ Other chromosomal abnormalities recurrently observed in MM samples (ie, 13q deletion, 1q gain/amplification, 17p deletion, and the HD status) appeared to be associated with less strong miRNA signatures, a finding that needs to be clarified in larger series.

Most of the 26 miRNAs significantly discriminating the TC groups have previously been found to be involved in solid and hematologic tumors. The most extensively investigated are $m i R-155, m i R-221$ and $m i R-222$, and the let-7 family. MiR-155 is involved in many biologic processes, including hematopoiesis, inflammation, and immunity, and its deregulation has been found to be associated with certain types of solid and hematologic tumors, in which it is predominantly overexpressed and acts as an oncomir. ${ }^{26}$ Notably, miR-155 has been very recently found deregulated in Waldenström macroglobulinemia (WM), suggesting a role in the proliferation and growth of WM cells acting on signaling cascades, including MAPK/ERK, PI3/AKT, and nuclear factor- $\kappa \mathrm{B}$ pathways. ${ }^{27} \mathrm{In}$ addition, $m i R-155$ knockdown leads to significant increase of WM cells in $\mathrm{G}_{1}$ phase and to the down-regulation of cyclin-dependent kinases and cyclins $\mathrm{D}$ and the simultaneous upregulation of $\mathrm{p} 53$ expression, suggesting a critical role in the regulation of cell-cycle proteins responsible for $\mathrm{G}_{1}$ arrest. ${ }^{27} M i R-221$ and $m i R-222$ have also been found to be up-modulated in many tumors and described to target the $C$-KIT, $p 27$, and $p 57$ genes. ${ }^{28,29}$ Finally, many human let-7 genes, which are known to target $R A S$ genes and oncogenes involved in the cell cycle, such as HMGA2, MYC, CDK6, and CDC25, ${ }^{30}$ map to regions frequently deleted in human tumors, indicating that they may function as tumor suppressors. The most striking finding was the very specific expression of 3 miRNAs ( $m i R-99 b$, let-7e, and miR-125a-5p), encoded in a conserved genomic cluster, in the $\mathrm{t}(4 ; 14)$ cases. They are coordinately up-regulated during metamorphosis in Drosophila, where they are cotranscribed as a single polycistronic transcript. ${ }^{31,32}$ Although their involvement has been suggested in various tumors, to our 
knowledge, this is the first evidence of their coordinated deregulation in cancer.

The differential miRNA expression associated with distinct genetic subgroups is a novel finding in MM. Of note, it has already been reported in other hematologic malignancies, such as acute myeloid leukemia ${ }^{33}$ and chronic lymphocytic leukemia. As regards this latter neoplasia, in which the role of miRNA has been extensively investigated, ${ }^{34}$ the presence of miRNA signatures associated with the major specific genetic lesions (trisomy 12 and $13 \mathrm{q} 14,11 \mathrm{q} 23$, and $17 \mathrm{p} 13$ deletions) has been reported very recently. ${ }^{35}$ Interestingly, as found in our study, the discriminating miRNAs were not localized in the chromosomal regions specific for the corresponding cytogenetic abnormalities. Overall, the identification of specific miRNA patterns may help not only to distinguish distinct MM genetic subgroups known to show differences in term of response to therapy and survival, but also to provide a better understanding of their pathogenesis.

We also evaluated the impact in miRNA expression of allelic imbalances occurring in MM. Our integrative analysis identified the occurrence of a gene dosage effect on the expression of several miRNAs, as has previously been reported in HMCLs. ${ }^{16}$ Interestingly, some of the miRNA genes map to hotspot-altered regions in MM: for example, mir-17 and mir-20a, which belong to a cluster at 13q31, which was deleted in almost $40 \%$ of our patients; furthermore, a number are located in odd-numbered chromosomes involved in hyperdiploidy, particularly chromosomes 3, 5, 7, 9, 11, and 19; and 3 (mir-1231, mir-205, and mir-215) belong to the long arm of chromosome 1, which was gained at a frequency of more than $30 \%$. Using the analog integrative approach, we evaluated the impact of $\mathrm{LOH}$ on miRNA expression, based on the data described in our recently published study. ${ }^{20}$ Among the identified miRNA whose expression correlated with the occurrence of $\mathrm{LOH}$, let-7b has been previously reported as the most discriminatory miRNA between acute lymphoid leukemia cases compared with acute myeloid leukemia cases (significantly down-regulated in acute lymphoid leukemia). ${ }^{36}$ Moreover, it has been demonstrated that let-7b correlated with the cytogenetic prognostic risk associated with the samples, being low in the favorable groups and high in intermediate and adverse acute myeloid leukemia cases. ${ }^{37}$ Of note, we also identified a significant correlation between $m i R-140-3 p$ expression and the occurrence of $\mathrm{LOH}$ at 16q22.1-q23.1, which has been described as recurrent in $\mathrm{MM}$ by others and us. ${ }^{20,38}$ We have also demonstrated that $\mathrm{LOH}$ at $16 \mathrm{q} 22.1$ significantly correlated with the expression of $W W P 2,{ }^{20}$ host gene of $m i R-140-3 p$ involved in ubiquitination processes, suggesting that LOH (associated with monoallelic loss or other mechanisms, such as uniparental disomy) may also represent an important mechanism in the coordinated control of miRNA and gene expression in MM.

The computational prediction of miRNA targets currently presents several significant challenges because all of the most widely used tools (miRanda, TargetScan, PicTar, PITA, and RNAhybrid) are characterized by a significant proportion of false-positive interactions $s^{39,40}$ that are partly because posttranscriptional regulation is context-dependent. On the basis of increasing experimental evidence supporting the hypothesis that miRNAs can act through target degradation, it has been proposed that target predictions could be integrated with miRNA and gene expression profiles to select functional miRNA/mRNA relationships. This can be done by adopting a variational Bayesian model and software, ${ }^{41,42}$ or simply using a nonheuristic method based on miRNA/mRNA anticorrelations. We applied the latter to our dataset, which allowed the reconstruction of a general miRNA/ mRNA regulatory network that represents the putative functional regulatory effects (as supported by expression data) of all of these miRNAs on their targets in MM.
On the basis of the target genes identified here, several the miRNAs differentially expressed in $I G H$ translocated cases may play important roles in the biology of MM PCs. With regard to the $t(4 ; 14)$ miRNA signature, 5 miRNAs target $C B F A 2 T 2$, a nuclear repressor homologous to ETO that binds to the AML1-ETO complex and may play a role in hematopoietic differentiation. ${ }^{43,44}$ Furthermore, let-7e targets PTPRE, a positive regulator of osteoclast function ${ }^{45}$ and a selective inhibitor of IL-6- and IL-10-induced JAK-STAT signaling. ${ }^{46}$ Interestingly, the expression of the tumor suppressor gene PDCD4 (programmed cell death 4), ${ }^{47}$ a supported target of $m i R-221$ based on our analysis, has recently been found to depend on the levels of MMSET, which is deregulated by the $\mathrm{t}(4 ; 14){ }^{48} I N G 4$, a tumor suppressor frequently mutated or down-regulated in human cancers, which was recently described to exert an inhibitory effect on MM-induced angiogenesis, ${ }^{49,50}$ is a supported target of miR-365. Concerning the TC5 signature, miR-133a targets DMTF1, a putative tumor suppressor that activates the ARF-p53 pathway, leading to cell growth arrest or apoptosis; notably, it maps at $7 \mathrm{q} 21$, often deleted in human malignancies. ${ }^{51}$ Finally, among the miRNAs up-regulated in $\mathrm{t}(11 ; 14), m i R-361-3 p$ and $m i R-30 e^{*}$ target $P P P 2 R 4$, an activator subunit of PP2A, which plays an important role in the survival and growth of MM cells because it dephosphorylates the GP130 subunit of the IL-6 receptor, thus preventing its degradation and allowing the activation of IL-6 signaling. 52,53

Taken together, our findings strongly suggest that understanding the molecular biology of myeloma requires considering the miRNome in the context of the genomic and transcriptomic features of malignant PCs. Based on this integrated approach, our data may provide an important contribution to future investigations aimed at characterizing the role of specific miRNAs in MM pathogenesis.

\section{Acknowledgments}

This work was supported by Associazione Italiana Ricerca sul Cancro (A.N.), Associazione Italiana contro le Leucemie Milano, Fondazione CARIPARO (Progetti Eccellenza 2006 and PhD 2008), MIUR (PRIN2007Y84HTJ and PRIN2007CHSMEB), University of Padova (CPDR074285/07), University of Modena (Finanziamento 2008), and Fondazione Cassa di Risparmio di Modena (Bando ricerca 2007). L.A. and K.T. are supported by Fellowships from Fondazione Italiana Ricerca sul Cancro.

\section{Authorship}

Contribution: M.L. performed the research, performed miRNA profiling and quantitative RT-PCR, analyzed the data, and wrote the manuscript; M.B. and G.S. generated and analyzed the microRNA/ mRNA network data; L.A. performed transcriptional, genotyping, statistical, and integrative analyses and wrote the manuscript; K.T. generated gene expression profiling data; L.M. generated genotyping data; S.F. and G.L.D. collected patient samples; L.L. designed research and revised the manuscript; and S. Bicciato, S. Bortoluzzi, and A.N. designed research and wrote the manuscript.

Conflict-of-interest disclosure: The authors declare no competing financial interests.

Correspondence: Antonino Neri, Department of Medical Sciences, University of Milan, F. Sforza 35, 20122 Milano, Italy; e-mail: antonino.neri@unimi.it. 


\section{References}

1. Fonseca R, Barlogie B, Bataille R, et al. Genetics and cytogenetics of multiple myeloma: a workshop report. Cancer Res. 2004;64(4):1546-1558.

2. Bergsagel PL, Kuehl WM. Molecular pathogenesis and a consequent classification of multiple myeloma. J Clin Oncol. 2005;23(26):6333-6338.

3. Hideshima T, Bergsagel PL, Kuehl WM, Anderson $\mathrm{KC}$. Advances in biology of multiple myeloma: clinical applications. Blood. 2004;104(3):607-618.

4. Agnelli L, Bicciato S, Mattioli M, et al. Molecular classification of multiple myeloma: a distinct transcriptional profile characterizes patients expressing CCND1 and negative for 14q32 translocations. J Clin Oncol. 2005;23(29):7296-7306.

5. Agnelli L, Fabris S, Bicciato S, et al. Upregulation of translational machinery and distinct genetic subgroups characterise hyperdiploidy in multiple myeloma. Br J Haematol. 2007;136(4):565-573.

6. Agnelli L, Bicciato S, Fabris S, et al. Integrative genomic analysis reveals distinct transcriptional and genetic features associated with chromosome 13 deletion in multiple myeloma. Haematologica. 2007;92(1):56-65.

7. Chng WJ, Kumar S, Vanwier S, et al. Molecular dissection of hyperdiploid multiple myeloma by gene expression profiling. Cancer Res. 2007; 67(7):2982-2989.

8. Fabris S, Ronchetti D, Agnelli L, et al. Transcriptional features of multiple myeloma patients with chromosome 1q gain. Leukemia. 2007;21(5): 1113-1116.

9. Shaughnessy JD Jr, Zhan F, Burington BE, et al. A validated gene expression model of high-risk multiple myeloma is defined by deregulated expression of genes mapping to chromosome 1. Blood. 2007;109(6):2276-2284.

10. Zhan F, Huang Y, Colla S, et al. The molecular classification of multiple myeloma. Blood. 2006; 108(6):2020-2028.

11. Bartel DP. MicroRNAs: genomics, biogenesis, mechanism, and function. Cell. 2004;116(2):281297.

12. Calin GA, Croce CM. MicroRNAs and chromosomal abnormalities in cancer cells. Oncogene. 2006;25(46):6202-6210.

13. Calin GA, Croce CM. MicroRNA-cancer connection: the beginning of a new tale. Cancer Res. 2006;66(15):7390-7394.

14. Loffler D, Brocke-Heidrich K, Pfeifer G, et al Interleukin-6 dependent survival of multiple myeloma cells involves the Stat3-mediated induction of microRNA-21 through a highly conserved enhancer. Blood. 2007;110(4):1330-1333.

15. Pichiorri F, Suh SS, Ladetto $M$, et al. MicroRNAs regulate critical genes associated with multiple myeloma pathogenesis. Proc Natl Acad Sci U S A. 2008;105(35):12885-12890.

16. Lionetti M, Agnelli L, Mosca L, et al. Integrative high-resolution microarray analysis of human myeloma cell lines reveals deregulated miRNA expression associated with allelic imbalances and gene expression profiles. Genes Chromosomes Cancer. 2009;48(6):521-531.

17. Ronchetti $\mathrm{D}$, Lionetti M, Mosca L, et al. An integrative genomic approach reveals coordinated expression of intronic miR-335, miR-342, and miR-561 with deregulated host genes in multiple myeloma. BMC Med Genomics. 2008;1:37.

18. Roccaro AM, Sacco A, Thompson B, et al. MicroRNAs $15 a$ and 16 regulate tumor proliferation in multiple myeloma. Blood. 2009;113(26):66696680.

19. Mattioli M, Agnelli L, Fabris S, et al. Gene expression profiling of plasma cell dyscrasias reveals molecular patterns associated with distinct IGH translocations in multiple myeloma. Oncogene. 2005;24(15):2461-2473.

20. Agnelli L, Mosca L, Fabris S, et al. A SNP microarray and FISH-based procedure to detect allelic imbalances in multiple myeloma: an integrated genomics approach reveals a wide gene dosage effect. Genes Chromosomes Cancer. 2009;48(7):603-614

21. Ferrari F, Bortoluzzi S, Coppe A, et al. Novel definition files for human GeneChips based on GeneAnnot. BMC Bioinformatics. 2007;8:446.

22. Krek A, Grun D, Poy MN, et al. Combinatorial microRNA target predictions. Nat Genet. 2005;37(5):495-500.

23. Griffiths-Jones S, Saini HK, van DS, Enright AJ miRBase: tools for microRNA genomics. Nucleic Acids Res. 2008;36(Database issue):D154-D158.

24. Gennarino VA, Sardiello M, Avellino R, et al. MicroRNA target prediction by expression analysis of host genes. Genome Res. 2009;19(3):481 490

25. Shannon P, Markiel A, Ozier O, et al. Cytoscape: a software environment for integrated models of biomolecular interaction networks. Genome Res. 2003;13(11):2498-2504.

26. Esquela-Kerscher A, Slack FJ. Oncomirs: microRNAs with a role in cancer. Nat Rev Cancer. 2006;6(4):259-269.

27. Roccaro AM, Sacco A, Chen C, et al. microRNA expression in the biology, prognosis, and therapy of Waldenstrom macroglobulinemia. Blood. 2009 113(18):4391-4402

28. Lotterman CD, Kent OA, Mendell JT. Functiona integration of microRNAs into oncogenic and tumor suppressor pathways. Cell Cycle. 2008; 7(16):2493-2499.

29. Visone R, Croce CM. MiRNAs and cancer. Am J Pathol. 2009;174(4):1131-1138.

30. Medina PP, Slack FJ. microRNAs and cancer: an overview. Cell Cycle. 2008;7(16):2485-2492.

31. Sempere LF, Sokol NS, Dubrovsky EB, Berger EM, Ambros V. Temporal regulation of microRNA expression in Drosophila melanogaster mediated by hormonal signals and broad-complex gene activity. Dev Biol. 2003;259(1):9-18.

32. Sokol NS, Xu P, Jan YN, Ambros V. Drosophila let-7 microRNA is required for remodeling of the neuromusculature during metamorphosis. Genes Dev. 2008;22(12):1591-1596.

33. Jongen-Lavrencic M, Sun SM, Dijkstra MK, Valk PJ, Lowenberg B. MicroRNA expression profiling in relation to the genetic heterogeneity of acute myeloid leukemia. Blood. 2008;111(10):5078 5085.

34. Calin GA, Croce CM. Chronic lymphocytic leukemia: interplay between non-coding RNAs and protein-coding genes. Blood. 2009;114(22):4761 4770.

35. Visone R, Rassenti LZ, Veronese A, et al. Karyotype specific microRNA signature in chronic lymphocytic leukemia. Blood. 2009;114(18):38723879

36. Mi S, Lu J, Sun M, et al. MicroRNA expression signatures accurately discriminate acute lymphoblastic leukemia from acute myeloid leukemia.
Proc Natl Acad Sci U S A. 2007;104(50):1997119976.

37. Dixon-Mclver A, East P, Mein CA, et al. Distinctive patterns of microRNA expression associated with karyotype in acute myeloid leukaemia. PLoS ONE. 2008;3(5):e2141.

38. Jenner MW, Leone PE, Walker BA, et al. Gene mapping and expression analysis of $16 \mathrm{q}$ loss of heterozygosity identifies WWOX and CYLD as being important in determining clinical outcome in multiple myeloma. Blood. 2007;110(9):3291 3300.

39. Didiano D, Hobert O. Molecular architecture of a miRNA-regulated 3' UTR. RNA. 2008;14(7): 1297-1317.

40. Grimson A, Farh KK, Johnston WK, GarrettEngele P, Lim LP, Bartel DP. MicroRNA targeting specificity in mammals: determinants beyond seed pairing. Mol Cell. 2007;27(1):91-105

41. Huang JC, Babak T, Corson TW, et al. Using expression profiling data to identify human microRNA targets. Nat Methods. 2007;4(12):1045-1049.

42. Huang JC, Morris QD, Frey BJ. Bayesian inference of microRNA targets from sequence and expression data. J Comput Biol. 2007;14(5):550-563.

43. Fracchiolla NS, Colombo G, Finelli P, Maiolo AT, Neri A. EHT, a new member of the MTG8/ETO gene family, maps on 20q11 region and is deleted in acute myeloid leukemias. Blood. 1998;92(9): 3481-3484.

44. Lindberg SR, Olsson A, Persson AM, Olsson I. The leukemia-associated ETO homologues are differently expressed during hematopoietic differentiation. Exp Hematol. 2005;33(2):189-198.

45. Chiusaroli R, Knobler H, Luxenburg C, et al. Tyrosine phosphatase epsilon is a positive regulator of osteoclast function in vitro and in vivo. Mol Biol Cell. 2004;15(1):234-244.

46. Tanuma N, Shima H, Nakamura K, Kikuchi K Protein tyrosine phosphatase epsilonC selectively inhibits interleukin-6- and interleukin-10induced JAK-STAT signaling. Blood. 2001;98(10): 3030-3034

47. Lankat-Buttgereit B, Goke R. The tumour suppressor Pdcd4: recent advances in the elucidation of function and regulation. Biol Cell. 2009; 101(6):309-317

48. Brito JL, Walker B, Jenner M, et al. MMSET deregulation affects cell cycle progression and adhesion regulons in $\mathrm{t}(4 ; 14)$ myeloma plasma cells. Haematologica. 2009;94(1):78-86.

49. Colla S, Tagliaferri S, Morandi F, et al. The new tumor-suppressor gene inhibitor of growth family member 4 (ING4) regulates the production of proangiogenic molecules by myeloma cells and suppresses hypoxia-inducible factor-1 alpha (HIF-1alpha) activity: involvement in myelomainduced angiogenesis. Blood. 2007;110(13): 4464-4475.

50. Kim S. HuntING4 new tumor suppressors. Cell Cycle. 2005;4(4):516-517.

51. Inoue K, Mallakin A, Frazier DP. Dmp1 and tumor suppression. Oncogene. 2007;26(30):4329-4335

52. Kang HS, Lee BS, Yang $Y$, et al. Roles of protein phosphatase 1 and 2A in an IL-6-mediated autocrine growth loop of human myeloma cells. Cell Immunol. 1996;168(2):174-183.

53. Mitsuhashi S, Shima H, Tanuma N, et al. Protein phosphatase type 2A, PP2A, is involved in degradation of gp130. Mol Cell Biochem. 2005;269(1): 183-187. 


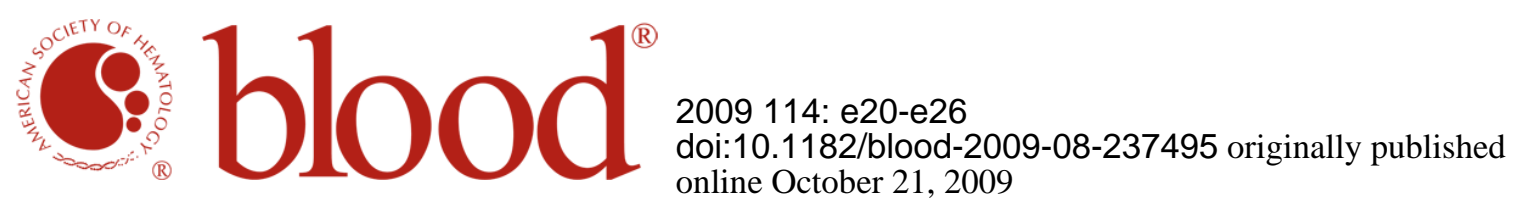

\section{Identification of microRNA expression patterns and definition of a microRNA/mRNA regulatory network in distinct molecular groups of multiple myeloma}

Marta Lionetti, Marta Biasiolo, Luca Agnelli, Katia Todoerti, Laura Mosca, Sonia Fabris, Gabriele Sales, Giorgio Lambertenghi Deliliers, Silvio Bicciato, Luigia Lombardi, Stefania Bortoluzzi and Antonino Neri

Updated information and services can be found at:

http://www.bloodjournal.org/content/114/25/e20.full.html

Articles on similar topics can be found in the following Blood collections

Information about reproducing this article in parts or in its entirety may be found online at:

http://www.bloodjournal.org/site/misc/rights.xhtml\#repub_requests

Information about ordering reprints may be found online at:

http://www.bloodjournal.org/site/misc/rights.xhtml\#reprints

Information about subscriptions and ASH membership may be found online at:

http://www.bloodjournal.org/site/subscriptions/index.xhtml

Blood (print ISSN 0006-4971, online ISSN 1528-0020), is published weekly by the American Society of Hematology, 2021 L St, NW, Suite 900, Washington DC 20036.

Copyright 2011 by The American Society of Hematology; all rights reserved. 